\title{
AN ERGODIC ACTION OF THE OUTER AUTOMORPHISM GROUP OF A FREE GROUP
}

\author{
WILLIAM M. GOLDMAN
}

\begin{abstract}
For $n>2$, the action of the outer automorphism group of the rank $n$ free group $F_{n}$ on $\operatorname{Hom}\left(F_{n}, \mathrm{SU}(2)\right) / \mathrm{SU}(2)$ is ergodic with respect to the Lebesgue measure class.
\end{abstract}

\section{INTRODUCTION}

Let $F_{n}$ be a free group of rank $n>1$ and let $G$ be a compact Lie group. Then $\operatorname{Hom}\left(F_{n}, G\right)$ admits a natural volume form which is invariant under $\operatorname{Aut}\left(F_{n}\right)$. This volume form descends to a finite measure on the character variety $\operatorname{Hom}\left(F_{n}, G\right) / G$ which is invariant under $\operatorname{Out}\left(F_{n}\right)$. The purpose of this note is to prove:

Theorem. Suppose that $G$ is a connected group locally isomorphic to a product of copies of $\mathrm{SU}(2)$ and $\mathrm{U}(1)$. If $n>2$, then the $\operatorname{Out}\left(F_{n}\right)$-action on $\operatorname{Hom}\left(F_{n}, G\right) / G$ is ergodic.

We conjecture that Out $\left(F_{n}\right)$ is ergodic on each connected component of $\operatorname{Hom}\left(F_{n}, G\right) / G$ for every compact Lie group $G$ and $n>2$.

When $G=\mathrm{U}(1)$, then this action is just the action of $\mathrm{GL}(n, \mathbb{Z})$ on the $n$-torus $\mathbb{R}^{n} / \mathbb{Z}^{n}$, which is well known to be ergodic. In fact, certain cyclic subgroups of $\mathrm{GL}(n, \mathbb{Z})$ act ergodicly.

The proof relies heavily on [2], both in its outline and a key result. When $n=2$, the action is not ergodic, since it preserves the function

$$
\begin{aligned}
\operatorname{Hom}\left(F_{n}, G\right) / G & \stackrel{\kappa}{\rightarrow}[-2,2] \\
{[\rho] } & \longmapsto \operatorname{tr}\left(\left[X_{1}, X_{2}\right]\right)
\end{aligned}
$$

where $X_{1}, X_{2}$ are a pair of free generators for $F_{2}$. However, for each $-2 \leq t \leq 2$, the action is ergodic on $\kappa^{-1}(t)$.

Date: August 21, 2018.

1991 Mathematics Subject Classification. 57M05 (Low-dimensional topology), 22D40 (Ergodic theory on groups).

Key words and phrases. character variety, free group, outer automorphism group, Nielsen transformation, ergodic equivalence relation.

The author gratefully acknowledges support from National Science Foundation grants DMS-0405605 and DMS-0103889 . 
When $\pi$ is the fundamental group of a closed surface, then Pickrell and Xia 4] have proved $\operatorname{Out}(\pi)$ is ergodic on $\operatorname{Hom}(\pi, G) / G$ for any compact Lie group $G$.

As in [2, the methods here apply when $G$ is any Lie group having simple factors $\mathrm{U}(1)$ and $\mathrm{SU}(2)$. In particular, since this class of groups is closed under the operation of taking direct products, the action of Out $\left(F_{n}\right)$ is ergodic on

$$
\operatorname{Hom}\left(F_{n}, G \times G\right) /(G \times G) \longleftrightarrow \operatorname{Hom}\left(F_{n}, G\right) / G \times \operatorname{Hom}\left(F_{n}, G\right) / G .
$$

As in [2], the action of $\operatorname{Out}\left(F_{n}\right)$ on $\operatorname{Hom}\left(F_{n}, G\right) / G$ is weak-mixing, that is:

Corollary. The only invariant finite-dimensional subrepresentation of the induced unitary representation of $\operatorname{Out}\left(F_{n}\right)$ on $L^{2}\left(\operatorname{Hom}\left(F_{n}, G\right) / G\right)$ consists of constants.

I would like to thank David Fisher for pointing out an error in the original proof of Lemma 3.1 and for many helpful suggestions.

\section{ERgodic theory of the SU(2)-CHARACTER VARIETy}

Let $\left\{X_{1}, \ldots, X_{n}\right\}$ be a set of free generators for $F_{n}$ and let

$$
X_{0}=X_{n}^{-1} \ldots X_{1}^{-1} \text {. }
$$

Then $F_{n}$ is the fundamental group of an $n+1$-holed sphere $S_{n+1}$, where the $X_{0}, X_{1}, \ldots, X_{n}$ correspond to components of $\partial S_{n+1}$. The mapping class group $\Gamma_{n+1}$ of $S_{n+1}$ embeds in $\operatorname{Out}\left(F_{n}\right)$ as the subgroup preserving the conjugacy classes of the cyclic subgroups $\left\langle X_{i}\right\rangle$ for $i=0, \ldots, n$.

The proof proceeds as follows. Let

$$
\operatorname{Hom}\left(F_{n}, G\right) / G \stackrel{f}{\rightarrow} \mathbb{R}
$$

be an Out $\left(F_{n}\right)$-invariant measurable function. We show that $f$ is constant almost everywhere.

The main result of [2] applied to the surface $S_{n+1}$ gives the following:

Proposition 1.1. The mapping

$$
\begin{aligned}
\operatorname{Hom}\left(F_{n}, G\right) / G \stackrel{t_{\partial}}{\longrightarrow}[-2,2]^{n+1} \\
{[\rho] \longmapsto\left[\begin{array}{c}
\operatorname{tr}\left(\rho\left(X_{0}\right)\right) \\
\vdots \\
\operatorname{tr}\left(\rho\left(X_{n}\right)\right)
\end{array}\right] }
\end{aligned}
$$

is an ergodic decomposition for the action of $\Gamma_{n+1}$. That is, for every $\Gamma_{n+1}$-invariant measurable function

$$
\operatorname{Hom}\left(F_{n}, G\right) / G \stackrel{h}{\rightarrow} \mathbb{R}
$$


there exists a measurable function

$$
[-2,2]^{n+1} \stackrel{H}{\rightarrow} \mathbb{R}
$$

such that $h=H \circ t_{\partial}$ almost everywhere.

Using the embedding of the mapping class group

$$
\Gamma_{n+1} \hookrightarrow \operatorname{Out}\left(F_{n}\right)
$$

as above, the Out $\left(F_{n}\right)$-invariant function $f$ is $\Gamma_{n+1}$-invariant, and hence factors through $t_{\partial}$.

By Proposition 1.1 there exists a function

$$
[-2,2]^{n+1} \stackrel{F}{\rightarrow} \mathbb{R}
$$

such that $f=F \circ t_{\partial}$, where $f$ is the function discussed in (1.1).

\section{THE CASE OF RANK $n=3$}

First consider the case $n=3$. Following the notation of [1, 2, denote the generators by

$$
A=X_{1}, \quad B=X_{2}, \quad C=X_{3}, \quad D=X_{0}
$$

so that $A, B, C, D$ are subject to the relation

$$
A B C D=1 .
$$

A representation $\rho$ is determined by its values

$$
(\rho(A), \rho(B), \rho(C)) \in G^{3}
$$

on the generators $A, B, C$ and

$$
\rho(D)=\rho(C)^{-1} \rho(B)^{-1} \rho(A)^{-1} \text {. }
$$

2.1. Trace coordinates. The equivalence class $[\rho]$ is determined by the seven functions

$$
\begin{aligned}
& a=\operatorname{tr}(\rho(A)) \\
& b=\operatorname{tr}(\rho(B)) \\
& c=\operatorname{tr}(\rho(C)) \\
& d=\operatorname{tr}(\rho(D))=\operatorname{tr}\left(\rho\left(D^{-1}\right)\right)=\operatorname{tr}(\rho(A B C)) \\
& x=\operatorname{tr}(\rho(A B)) \\
& y=\operatorname{tr}(\rho(B C)) \\
& z=\operatorname{tr}(\rho(C A))
\end{aligned}
$$


subject to the polynomial relation

$$
\begin{aligned}
x^{2}+y^{2}+z^{2}+x y z & = \\
(a b+c d) x & +(a d+b c) y+(a c+b d) z \\
& +\left(4-a^{2}-b^{2}-c^{2}-d^{2}-a b c d\right) .
\end{aligned}
$$

In other words, the $\mathrm{SL}(2, \mathbb{C})$-character variety of $F_{3}$ is the hypersurface in $\mathbb{C}^{7}$ defined by (2.2).

When $a, b, c, d \in \mathbb{R}$ the topology of the set of $\mathbb{R}$-points is analyzed in 11. In particular the $\mathbf{S U}(2)$-character variety is the union over the set $V$ of all

$$
(a, b, c, d) \in[-2,2]^{4}
$$

satisfying

$$
\begin{aligned}
0 \geq \Delta(a, b, c, d)= & \left(2\left(a^{2}+b^{2}+c^{2}+d^{2}\right)-a b c d-16\right)^{2} \\
& -\left(4-a^{2}\right)\left(4-b^{2}\right)\left(4-c^{2}\right)\left(4-d^{2}\right)
\end{aligned}
$$

of compact components of the cubic surface in $\mathbb{R}^{3}$ satisfying (2.2). Here is an alternate description with which it is easier to work.

2.2. Rank two free groups. The $\mathrm{SU}(2)$-character variety of $F_{2}$ is the subset $V_{3} \subset \mathbb{R}^{3}$ defined by traces

$$
\left(x_{1}, x_{2}, x_{3}\right) \in[-2,2]^{3}
$$

satisfying the inequality

$$
x_{1}^{2}+x_{2}^{2}+x_{3}^{2}+x_{1} x_{2} x_{3} \leq 4
$$

which is depicted in Figure 11.

A quadruple $(a, b, c, d) \in[-2,2]^{4}$ is the image of an $\mathrm{SU}(2)$-character if and only if there exists $y \in \mathbb{R}$ such that both triples $(a, d, y)$ and $(b, c, y)$ lie in $V_{3}$.

Using (2.2), we determine the condition that $(a, d, y) \in V_{3}$. Apply (2.2) to $x_{1}=y, x_{2}=a, x_{3}=d$ to see that $(a, d, y) \in V_{3}$ if and only if $y$ lies in the interval

$$
Y(a, d):=\left[y_{-}(a, d), y_{+}(a, d)\right]
$$

with endpoints

$$
y_{ \pm}(a, d):=\frac{a d \pm \sqrt{\left(4-a^{2}\right)\left(4-d^{2}\right)}}{2} .
$$


For any $(a, d) \in[-2,2]^{2}$, the interval $Y(a, d)$ is nonempty, so that the restriction of the projection

$$
V_{3} \stackrel{\Pi_{a, d}}{\longrightarrow}[-2,2]^{2}
$$

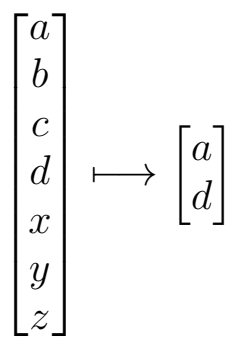

is onto. Furthermore the fiber $V_{3}(a, d)$ of the surjection

$$
\begin{gathered}
t_{\partial}\left(V_{3}\right) \stackrel{\Pi_{a, d}}{\rightarrow}[-2,2]^{2} \\
{\left[\begin{array}{l}
a \\
b \\
c \\
d
\end{array}\right] \longmapsto\left[\begin{array}{l}
a \\
d
\end{array}\right]}
\end{gathered}
$$

consists of all $(b, c) \in[-2,2]^{2}$ such that

$$
Y(b, c) \cap Y(a, d) \neq \emptyset \text {. }
$$

If $-2<y<2$, then the set of $(b, c)$ such that $y \in Y(b, c)$ is the closed elliptical region $\overline{\mathcal{E}}_{y}$ inscribed in the square $[-2,2]^{2}$ at the four points

$$
(2, y),(y, 2),(-2,-y),(-y, 2) \text {, }
$$

depicted in Figure 3. If $y= \pm 2$, then the set of $(b, c)$ such that $y \in$ $Y(b, c)$ is the line segment

$$
\{(b, \mp b) \mid-2 \leq b \leq 2\} .
$$

For fixed $a, d \in[-2,2]$, the fiber $\Pi^{-1}\left(V_{3}(a, d)\right)$ equals

$$
\bigcup_{y \in Y(a, d)}\{(b, c) \mid y \in Y(b, c)\}
$$

depicted in Figure 4 


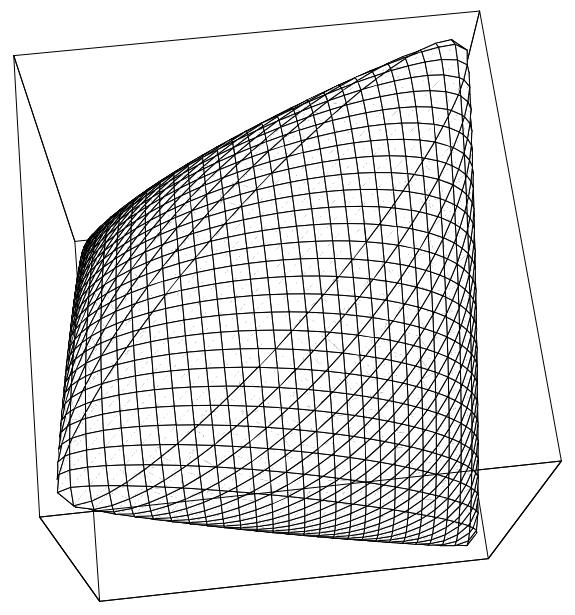

Figure 1. The SU(2)-character variety of a rank two free group is the region inside this surface. The surface, a rounded tetrahedron, consists of characters of abelian representations. It is a quotient of the 2-torus by an involution, where the fixed points of the involution correspond to the vertices of the tetrahedron.

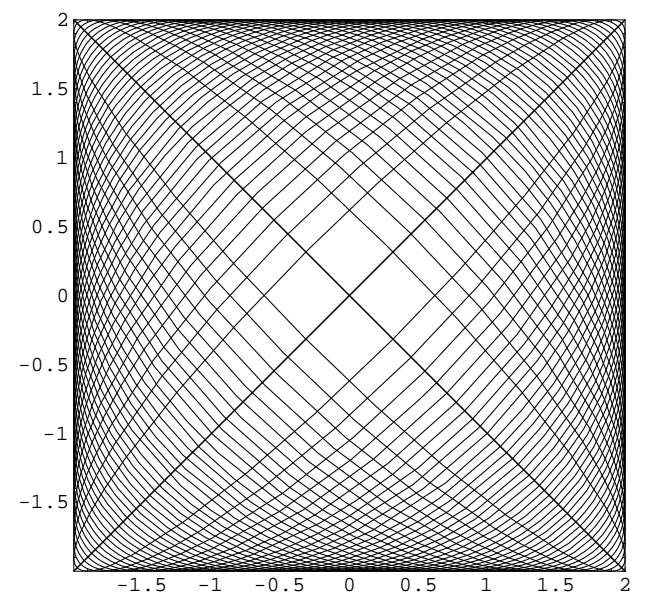

Figure 2. The SU(2)-character variety has three foliations by ellipses, corresponding to the three coordinate planes. This figure depicts projections of the leaves of one of the foliations into a coordinate plane. Leaves of the other two foliations project to to horizontal and vertical line segments, respectively. 


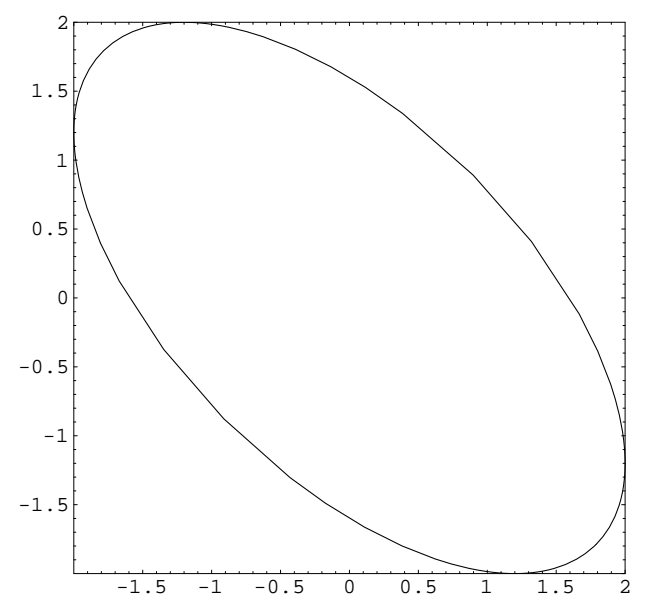

Figure 3. For $y_{0}=-1.2$, the set of possible $(b, c) \in$ $[-2,2]^{2}$ for which $\left(b, c, y_{0}\right)$ is the character of an $\mathrm{SU}(2)$ representation is the interior of the ellipse $\mathcal{E}_{1.2}$ inscribed in $b[-2,2]^{2}$ at the four points $( \pm 2, \mp 1.2)$ and $(\mp 1.2, \pm 2)$.

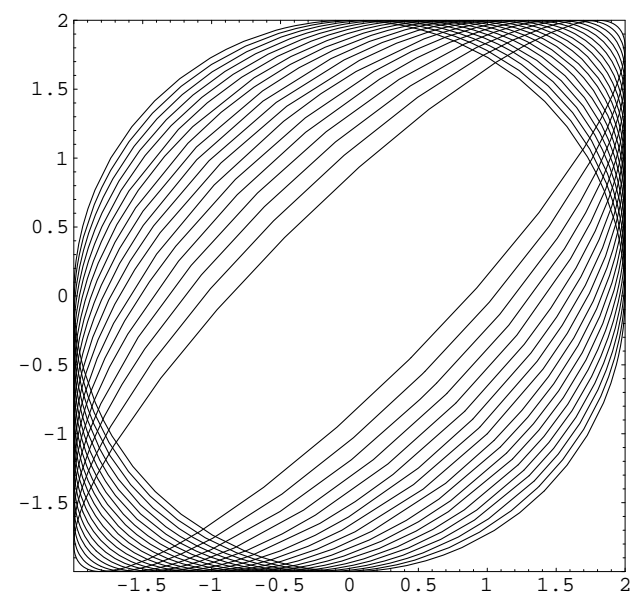

FiguRE 4. Here are the ellipses drawn for the interval $Y=[0,1.8]$. The union of the closed elliptical regions is the $(b, c)$-projection of the $V_{4}(a, d)$ where $a d=y_{+} y_{+}=1.8$ and $a^{2}+d^{2}=\left(\right.$ since $\left.y_{-}=0\right)$, for example $a=1.69, d=1.062$. 
2.3. A non-geometric automorphism. The automorphism $\alpha \in \operatorname{Aut}\left(F_{3}\right)$ defined by:

$$
\begin{aligned}
& A \stackrel{\alpha}{\longmapsto} A \\
& B \stackrel{\alpha}{\longmapsto} B A^{-1} \\
& C \stackrel{\alpha}{\longmapsto} A C \\
& D \stackrel{\alpha}{\longmapsto} D
\end{aligned}
$$

induces the following automorphism of the character variety:

$$
\left[\begin{array}{l}
a \\
b \\
c \\
d \\
x \\
y \\
z
\end{array}\right] \stackrel{\alpha^{*}}{\longmapsto}\left[\begin{array}{c}
a \\
x \\
a c-z \\
d \\
a x-b \\
y \\
c
\end{array}\right]
$$

Since $\alpha^{*}$ preserves the coordinates $a, d, y$, this automorphism restricts to a diffeomorphism on the level sets $a=a_{0}, d=d_{0}$ and $y=y_{0}$ and the restriction is linear:

$$
\left[\begin{array}{l}
x \\
b \\
c \\
z
\end{array}\right] \stackrel{\alpha^{*}}{\longmapsto}\left[\begin{array}{cccc}
a_{0} & -1 & 0 & 0 \\
1 & 0 & 0 & 0 \\
0 & 0 & a_{0} & -1 \\
0 & 0 & 1 & 0
\end{array}\right]\left[\begin{array}{l}
x \\
b \\
c \\
z
\end{array}\right]
$$

The following elementary fact (whose proof is omitted) is useful:

Lemma 2.1. Let $-2<a<2$. The linear transformation

$$
\begin{aligned}
\mathbb{R}^{2} \stackrel{L_{a}}{\longmapsto} \mathbb{R}^{2} \\
{\left[\begin{array}{l}
x \\
y
\end{array}\right] \longmapsto\left[\begin{array}{c}
a x-y \\
x
\end{array}\right] }
\end{aligned}
$$

preserves the positive definite quadratic form

$$
\left[\begin{array}{l}
x \\
y
\end{array}\right] \stackrel{Q}{\longmapsto} x^{2}-a x y+y^{2}
$$

and lies in the linear flow generated by the vector field

$$
\Upsilon:=\left(\frac{a}{2} x-y\right) \partial_{x}+\left(x-\frac{a}{2} y\right) \partial_{y} .
$$


The trajectories of $\Upsilon$ are the level sets of $Q$, which are ellipses. $L_{a}$ is linearly conjugate to a rotation by angle

$$
\theta=2 \cos ^{-1}(a / 2) .
$$

If $\theta \notin \pi \mathbb{Q}$, then $L_{a}$ has infinite order and is ergodic on each $Q$-level set.

The 4-dimensional affine subspaces of $\mathbb{R}^{7}$ corresponding to levels of $\left(a_{0}, d_{0}, y_{0}\right)$ split as products of two affine 2-planes (corresponding to levels of $(x, b)$ and $(c, z)$ respectively). Evidently the linear map $\alpha^{*}$ on these 4-planes splits as a direct sum of two copies of the linear map $L_{a_{0}}$. It preserves the trajectories of the linear vector field

$$
\begin{aligned}
\mathcal{A}=( & \left.\frac{a_{0}}{2} x-b\right) \partial_{x}+\left(x-\frac{a_{0}}{2} b\right) \partial_{b} \\
& +\left(\frac{a_{0}}{2} c-z\right) \partial_{c}+\left(c-\frac{a_{0}}{2} z\right) \partial_{z} .
\end{aligned}
$$

The zeroes of this vector field consist of the origin

$$
\left[\begin{array}{l}
x \\
b \\
c \\
z
\end{array}\right]=\left[\begin{array}{l}
0 \\
0 \\
0 \\
0
\end{array}\right]
$$

and, when $a_{0}= \pm 2$, the squares defined by

$$
x= \pm b, \quad z= \pm c .
$$

All other trajectories are ellipses. The transformation $\alpha^{*}$ acts by rotation along these ellipses through angle $\theta$ given by (2.4). When $\theta / \pi$ is irrational, this action is ergodic. Thus, for almost every $a_{0} \in[-2,2]$, the restriction of $\alpha^{*}$ to these ellipses is ergodic. On a set of full measure, the function $f$ is constant along the projections of trajectories of $\mathcal{A}$.

Fix $\left(a_{0}, d_{0}\right) \in(-2,2)^{2}$ and consider the equivalence relation $\sim$ on $V_{3}\left(a_{0}, d_{0}\right)$ generated by the projections of trajectories of $\mathcal{A}$.

Lemma 2.2. For $a_{0}, d_{0} \neq \pm 2$, all points in $V_{3}\left(a_{0}, d_{0}\right)$ are $\sim$-equivalent.

Proof. Since $V_{3}\left(a_{0}, d_{0}\right)$ is connected, it suffices to prove that each equivalence class is open. To this end, suppose that $\left(b_{0}, c_{0}\right) \in V_{3}\left(a_{0}, d_{0}\right)$; we show that every $(b, c)$ sufficiently close to $\left(b_{0}, c_{0}\right)$ is equivalent to $\left(b_{0}, c_{0}\right)$. 
The image of the tangent vector $\mathcal{A}$ at $(x, b, c, z)$ under the differential of the coordinate projection

$$
\left[\begin{array}{l}
x \\
b \\
c \\
z
\end{array}\right] \stackrel{\Pi_{b, c}}{\longmapsto}\left[\begin{array}{l}
b \\
c
\end{array}\right]
$$

is

$$
\left(\Pi_{b, c}\right)_{*} \mathcal{A}=\left(x-\frac{a_{0}}{2} b\right) \partial_{b}+\left(\frac{a_{0}}{2} c-z\right) \partial_{c}
$$

The fiber $\Pi_{b, c}^{-1}\left(b_{0}, c_{0}\right)$ is an interval. For some (and hence almost every) $(x, z) \in \Pi_{b, c}^{-1}\left(b_{0}, c_{0}\right)$, the vector

$$
\left(\Pi_{b, c}\right)_{*} \mathcal{A}\left(b_{0}, x, z, c_{0}\right)
$$

is nonzero. Choose such an $\left(x_{0}, z_{0}\right)$. For any open neighborhood $U$ of $\left(x_{0}, z_{0}\right)$, the values $\left(\Pi_{b, c}\right)_{*} \mathcal{A}\left(b_{0}, x, z, c_{0}\right)$ for $(x, z) \in U$, span $\mathbb{R}^{2}$.

Let $\Phi_{t}$ denote the flow generated by $\mathcal{A}$ and choose an open neighborhood $U_{0}$ of $\left(b_{0}, x_{0}, z_{0}, c_{0}\right)$ in $V_{3}\left(a_{0}, d_{0}\right)$. The differential of the map

$$
\begin{aligned}
\mathbb{R} \times\left(\left\{b_{0}\right\} \times U_{0} \times\left\{c_{0}\right\}\right) & \longrightarrow \mathbb{R}^{2} \\
\left(t, b_{0}, x, z, c_{0}\right) & \longmapsto \Pi_{b, c}\left(\Phi_{t}\left(b_{0}, x, z, c_{0}\right)\right)
\end{aligned}
$$

at $\left(0, b_{0}, x_{0}, z_{0}, c_{0}\right)$ is onto. The inverse function theorem guarantees an open neighborhood of $\left(0, b_{0}, x_{0}, z_{0}, c_{0}\right)$ mapping onto an open neighborhood of $\left(b_{0}, c_{0}\right)$, as desired.

Thus, for almost every $\left(a_{0}, d_{0}\right) \in[-2,2]^{2}$, the function $F$ of (1.2) is constant along the level surfaces $\Pi_{a, d}^{-1}\left(a_{0}, d_{0}\right)$, and hence factors through the projection $\Pi_{a, d}$ :

$$
F(a, b, c, d)=F(a, d) .
$$

Applying the same argument to the automorphism

$$
\begin{aligned}
& A \stackrel{\gamma}{\longmapsto} C A \\
& B \stackrel{\gamma}{\longmapsto} B \\
& C \stackrel{\gamma}{\longmapsto} C \\
& D \stackrel{\gamma}{\longmapsto} D C^{-1}
\end{aligned}
$$

implies that $F$ factors through the projection $\Pi_{(b, c)}$ and $F$ is almost everywhere constant. 
Thus the function $f$, which is invariant under the mapping class group $\Gamma_{4}$ and the automorphisms $\alpha_{*}, \gamma_{*}$, must be constant almost everywhere. This completes the proof of the Theorem when $n=3$.

\section{General RANK}

The case of rank $n>3$ follows easily from the case $n=3$. The following elementary lemma is useful:

Lemma 3.1. Let $G$ be a compact Lie group. Then $\operatorname{Out}\left(F_{n}\right)$ is ergodic on $\operatorname{Hom}\left(F_{n}, G\right) / G$ if and only if $\operatorname{Aut}\left(F_{n}\right)$ is ergodic on $\operatorname{Hom}\left(F_{n}, G\right)$. More generally, let

$$
\operatorname{Hom}\left(F_{n}, G\right) \stackrel{\Pi}{\rightarrow} \operatorname{Hom}\left(F_{n}, G\right) / G
$$

denote the quotient map and suppose $\Gamma \subset \operatorname{Aut}\left(F_{n}\right)$ is a subgroup containing $\operatorname{lnn}\left(F_{n}\right)$. A measurable $\Gamma$-invariant mapping.

$$
\operatorname{Hom}\left(F_{n}, G\right) / G \stackrel{f}{\rightarrow} W
$$

is an ergodic decomposition for $\Gamma$ if and only if

$$
\operatorname{Hom}\left(F_{n}, G\right) \stackrel{f \circ \Pi}{\longrightarrow} W
$$

is an ergodic decomposition for $\Gamma$.

Proof. For almost every $\rho \in \operatorname{Hom}\left(F_{n}, G\right)$, the image $\rho\left(F_{n}\right)$ is dense in $G$. Thus $\operatorname{Inn}\left(F_{n}\right)$ is ergodic on each $G$-orbit in $\operatorname{Hom}\left(F_{n}, G\right)$, and the quotient map $\Pi$ is an ergodic decomposition for the $\operatorname{lnn}\left(F_{n}\right)$-action. Since $\operatorname{lnn}\left(F_{n}\right) \subset \Gamma$, every $\Gamma$-invariant function

$$
\operatorname{Hom}\left(F_{n}, G\right) \stackrel{h}{\rightarrow} W
$$

(where $W$ is a standard measure space) factors through $\Pi$. Thus composition with $\Pi$ induces an isomorphism between the set of equivalence classes of $\Gamma$-invariant measurable maps on $\operatorname{Hom}\left(F_{n}, G\right)$ and on $\operatorname{Hom}\left(F_{n}, G\right) / G$. In particular the ergodic decompositons for the respective $\Gamma$-actions are $\Pi$-related, as desired.

In the following lemma, let $\iota_{j}$ (for $\left.j=1, \ldots, n\right)$ denote the monomorphism defined by

$$
\begin{aligned}
F_{n-1} & \stackrel{\iota_{j}}{\hookrightarrow} F_{n} \\
X_{i} & \longmapsto \begin{cases}X_{i} & \text { if } i<j \\
X_{i+1} & \text { if } i \geq j\end{cases}
\end{aligned}
$$

for $i=1, \ldots, n-1$. Denote by

$$
\operatorname{Aut}\left(F_{n-1)} \stackrel{I_{j}}{\hookrightarrow} \operatorname{Aut}\left(F_{n}\right)\right.
$$


the homomorphism defined by

$$
X_{i} \stackrel{I_{j}(\phi)}{\longmapsto} \begin{cases}X_{j} & \text { if } i=j \\ \iota_{j}\left(\phi\left(\iota_{j}^{-1}\left(X_{i}\right)\right)\right) & \text { if } i \neq j\end{cases}
$$

for $\phi \in \operatorname{Aut}\left(F_{n}\right)$. Clearly $\iota_{j}$ is equivariant with respect to $I_{j}$.

Lemma 3.2. Let $n>3$. Suppose that $\operatorname{Aut}\left(F_{n-1}\right)$ is ergodic on $\operatorname{Hom}\left(F_{n-1}, G\right)$. Let $1 \leq j \leq n$. Then

$$
\begin{aligned}
\operatorname{Hom}\left(F_{n}, G\right) & \stackrel{t_{j}}{\longrightarrow}[-2,2] \\
\rho & \longmapsto \operatorname{tr}\left(\rho\left(X_{j}\right)\right)
\end{aligned}
$$

is an ergodic decomposition for the action of $I_{j}\left(\operatorname{Aut}\left(F_{n-1}\right)\right) \times \operatorname{lnn}(G)$.

Proof. Clearly $t_{j}$ is $I_{j}\left(\operatorname{Aut}\left(F_{n-1}\right)\right.$-invariant. Let $-2<\tau<2$. The fiber $t_{j}^{-1}(\tau)$ identifies with the set of equivalence classes of

$$
\left(\left(\rho\left(X_{1}\right), \ldots, \rho\left(X_{n-1}\right), \rho\left(X_{n}\right)\right)\right.
$$

where $\operatorname{tr}\left(\rho\left(X_{j}\right)\right)=\tau$, that is, the set of $\operatorname{lnn}(G)$-orbits of

$$
\operatorname{Hom}\left(F_{n-1}, G\right) \times \operatorname{tr}^{-1}(\tau) \text {. }
$$

By Lemma 3.1 and the ergodicity hypothesis, Aut $\left(F_{n-1}\right)$ is ergodic on $\operatorname{Hom}\left(F_{n-1}, G\right)$. Transitivity of the $\operatorname{Inn}(G)$-action on $\operatorname{tr}^{-1}(\tau)$ implies ergodicity of Aut $\left(F_{n-1}\right) \times \operatorname{lnn}(G)$ acting on the subset of $\operatorname{Hom}\left(F_{n}, G\right)$ corresponding to $t_{j}^{-1}(\tau)$, which is equivalent to ergodicity of $\operatorname{Out}\left(F_{n-1}\right)$ on $t_{j}-1(\tau)$.

Suppose inductively that $\operatorname{Aut}\left(F_{n-1}\right)$ is ergodic on $\operatorname{Hom}\left(F_{n-1}, G\right)$. By Lemma 3.2. any $I_{j}\left(\operatorname{Aut}\left(F_{n-1}\right)\right)$-invariant measurable function $f$ factors through $t_{j}$ for each $j=1, \ldots, n$. We need only consider $j=1$ and $j=n$. Since

$$
\operatorname{Hom}\left(F_{n}, G\right) / G \stackrel{\left(t_{1}, t_{n}\right)}{\longrightarrow}[-2,2]^{2}
$$

is onto, any two points $\rho, \rho^{\prime}$ in a full measure subset of $\operatorname{Hom}\left(F_{n}, G\right)$ can be joined by a third $\rho^{\prime \prime}$ such that

$$
\begin{aligned}
& t_{1}\left(\rho^{\prime \prime}\right)=t_{1}(\rho), \\
& t_{n}\left(\rho^{\prime \prime}\right)=t_{n}\left(\rho^{\prime}\right) .
\end{aligned}
$$

Let $f$ be an $\operatorname{Aut}\left(F_{n}\right)$-invariant measurable function on $\operatorname{Hom}\left(F_{n}, G\right) / G$. Factorization of $f$ through $t_{1}$ implies that

$$
f(\rho)=f\left(\rho^{\prime \prime}\right)
$$

and factorization of $f$ through $t_{n}$ implies that

$$
f\left(\rho^{\prime \prime}\right)=f\left(\rho^{\prime}\right) .
$$


Thus $f(\rho)=f\left(\rho^{\prime \prime}\right)$, and $f$ is constant almost everywhere, as desired.

\section{Other LiE Groups}

The extension of the proof to products of $\mathrm{SU}(2)$ and $\mathrm{U}(1)$ proceeds exactly as in [2]. Away from a null subset of $\operatorname{Hom}\left(F_{n}, G\right) / G$, the action of the automorphisms of $F_{n}$ preserve tori (products of the $\mathrm{U}(1)$ factors and the invariant ellipses). Furthermore on almost every torus, the action is ergodic, and hence every invariant function factors through the trace functions of the generators. The rest of the proof is completely analogous.

\section{REFERENCES}

1. R. Benedetto and W. Goldman, The topology of the relative character variety of the quadruply-punctured sphere, Experimental Mathematics (1999), no. 8:1, $85-104$.

2. W. Goldman, Ergodic theory on moduli spaces, Ann. Math. (1997), no. 146, $475-507$.

3. - Action of the modular group on real SL(2)-characters of a one-holed torus, Geometry and Topology. (2003), no. 7, 443-486.

4. D. Pickrell and E. Xia, Ergodicity of mapping class group actions on representation varieties, I. Closed surfaces, Comment. Math. Helv. (2001), no. 77, 339-362.

Department of Mathematics, University of Maryland, College Park, MD 20742

E-mail address: wmg@@math.umd.edu 